\title{
AN ESTIMATOR OF THE NUMBER OF COMPONENTS OF A FINITE MIXTURE OF MULTIVARIATE DISTRIBUTIONS
}

\author{
Jogi Henna*
}

\begin{abstract}
An estimator of the number of components of a finite mixture of $k$-dimensional distributions is given on the basis of a one-dimensional independent random sample obtained by a transformation of a $k$-dimensional independent random sample. Some properties of the estimator are given. Some simulation results also are given for the case of finite mixtures of two-dimensional normal distributions.
\end{abstract}

Key words and phrases: $k$-dimensional finite mixture, normal pdf, number of components, one-dimensional finite mixture.

\section{Introduction}

Let $\mathcal{R}^{\ell}$ be an $\ell$-dimensional Euclidean space. Consider a family $\mathcal{F}=\left\{f_{\boldsymbol{\theta}}(\boldsymbol{x})\right.$ : $\boldsymbol{\theta} \in \Theta\}$ of known $k$-dimensional probability density functions (pdf's), where the parameter space $\Theta$ is a compact subset of $\mathcal{R}^{d_{1}}$ for a $d_{1}$. For a positive integer $m$, a pdf given by

$$
f\left(\boldsymbol{x} \mid \mathcal{A}_{m}^{\circ}\right)=\sum_{i=1}^{m} p_{i}^{\circ} f_{\boldsymbol{\theta}_{i}^{\circ}}(\boldsymbol{x})
$$

is called a finite mixture of $f_{\boldsymbol{\theta}_{1}^{\circ}}(\boldsymbol{x}), f_{\boldsymbol{\theta}_{2}^{\circ}}(\boldsymbol{x}), \ldots, f_{\boldsymbol{\theta}_{m}^{\circ}}(\boldsymbol{x})$ (Titterington et al. (1985)), where $\mathcal{A}_{m}^{\circ}=\left(p_{1}^{\circ}, p_{2}^{\circ}, \ldots, p_{m}^{\circ} ; \boldsymbol{\theta}_{1}^{\circ}, \boldsymbol{\theta}_{2}^{\circ}, \ldots, \boldsymbol{\theta}_{m}^{\circ}\right)$ with $\sum_{i=1}^{m} p_{i}^{\circ}=1,0<p_{i}^{\circ} \leq 1$ and $\boldsymbol{\theta}_{i}^{\circ} \in \Theta(i=1,2, \ldots, m)$. So a single $f_{\boldsymbol{\theta}}(\boldsymbol{x})$ in $\mathcal{F}$ is also considered a finite mixture for $m=1$ as a special case. Each $f_{\boldsymbol{\theta}_{i}^{\circ}}(\boldsymbol{x})$ is called a component of $f\left(\boldsymbol{x} \mid \mathcal{A}_{m}^{\circ}\right)$ and each $p_{i}^{\circ}$ a mixing ratio of $f_{\boldsymbol{\theta}_{i}^{\circ}}(\boldsymbol{x})$.

The purpose of this paper is to give an estimator $\widehat{m}_{n}$ of the number $m$ of components on the basis of an independent random sample $\left(\boldsymbol{X}_{1}, \boldsymbol{X}_{2}, \ldots, \boldsymbol{X}_{n}\right)$ from the distribution (1.1). The importance to estimate the number $m$ is described in McLachlan and Basford (1988), Titterington (1990) and others. Henna (1985), Feng and McCulloch (1994), Chen and Kalbfleisch (1996) and Richardson and Green (1997) have treated one-dimensional finite mixtures. Roeder (1994) has investigated a graphical technique to determine the number of components in a case of normal mixture, and Keribin (2000) has given a method which can be applied to a special type of multivariate normal mixture under the assumption that a superior value $Q$ of $m$ is known. Methods to determine the number of components are described in McLachlan and Peel (2000). Chen et al. (2001) and Garel (2001) have given a test for $m$ in a univariate case.

Received June 5, 2006. Revised August 26, 2006. Accepted December 7, 2006.

*Faculty of Science, University of the Ryukyus, 1 Senbaru, Nishihara-cho, Okinawa 903-0213, Japan. 
In this paper, a method which can be applied to $k$-dimensional finite mixture distributions is considered, however the analysis is based on one-dimensional samples. An estimator which can be considered an improvement of that of Henna (2005) is given. To attain our objectives, we find a column vector $\boldsymbol{a}$ and a scalar $\rho$ which satisfy the following condition, that is, putting $Y_{j}=\boldsymbol{a}^{\prime} \boldsymbol{X}_{j}+\rho$ $(j=1,2, \ldots, n)$, then $\left(Y_{1}, Y_{2}, \ldots, Y_{n}\right)$ can be regarded as an independent random sample from a finite mixture with $m$ components such as

$$
h\left(y \mid \boldsymbol{c}_{m}^{\circ}\right)=\sum_{i=1}^{m} p_{i}^{\circ} h_{\boldsymbol{\delta}_{i}^{\circ}}(y),
$$

where $h_{\boldsymbol{\delta}_{i}^{\circ}}(y)$ is a one-dimensional pdf with a parameter $\boldsymbol{\delta}_{i}^{\circ}$ and $\boldsymbol{c}_{m}^{\circ}=\left(p_{1}^{\circ}, p_{2}^{\circ}, \ldots\right.$, $\left.p_{m}^{\circ} ; \boldsymbol{\delta}_{1}^{\circ}, \boldsymbol{\delta}_{2}^{\circ}, \ldots, \boldsymbol{\delta}_{m}^{\circ}\right)$. In other words, by transforming $\left(\boldsymbol{X}_{1}, \boldsymbol{X}_{2}, \ldots, \boldsymbol{X}_{n}\right)$, we obtain an independent random sample $\left(Y_{1}, Y_{2}, \ldots, Y_{n}\right)$ which can be considered to have come from a finite mixture of $m$ one-dimensional distributions, and then construct an estimator $\widehat{m}_{n}$ on the basis of $\left(Y_{1}, Y_{2}, \ldots, Y_{n}\right)$.

In Section 2, some notations and preliminary lemmas are given. In Section 3, an estimator $\widehat{m}_{n}$ is constructed and it is shown that $\widehat{m}_{n}=m$ holds with probability one for $n$ sufficiently large. In Section 4 , it is shown that $\widehat{m}_{n}$ is independent of a norm $\|\boldsymbol{a}\|$. In Section 5, results similar to those of Sections 3 and 4 are given when $\mathcal{F}$ is a known finite family. In Section 6 , simulation results are given. In Section 7 , final discussions are provided.

\section{Notations and preliminary lemmas}

Assume that $Y=\boldsymbol{a}^{\prime} \boldsymbol{X}+\rho(\boldsymbol{a} \neq \mathbf{0})$ has a one-dimensional pdf $h_{\boldsymbol{\delta}}(y)$ with a parameter $\boldsymbol{\delta} \in \mathcal{R}^{d_{2}}$ for a $d_{2}$ when $\boldsymbol{X}$ has $f_{\boldsymbol{\theta}}(\boldsymbol{x})$ and that the parameter space given by

$$
\Delta(\boldsymbol{a}, \rho)=\{\boldsymbol{\delta}: \boldsymbol{\theta} \in \Theta\}
$$

is a compact subset of $\mathcal{R}^{d_{2}}$, where $\boldsymbol{\theta}$ corresponds to $\delta$ through $Y=\boldsymbol{a}^{\prime} \boldsymbol{X}+\rho$. The correspondence of $\Theta$ to $\Delta(\boldsymbol{a}, \rho)$ is not necessarily needed to be one-to-one. The following can be easily obtained (Billingsley (1986)).

Lemma 2.1. Assume that $\boldsymbol{X}$ has the finite mixture (1.1), then $Y=\boldsymbol{a}^{\prime} \boldsymbol{X}+\rho$ has the finite mixture (1.2) provided that some of $\boldsymbol{\delta}_{1}^{\circ}, \boldsymbol{\delta}_{2}^{\circ}, \ldots, \boldsymbol{\delta}_{m}^{\circ}$ may be equal, where $\boldsymbol{\theta}_{i}^{\circ}$ corresponds to $\boldsymbol{\delta}_{i}^{\circ}(i=1,2, \ldots, m)$.

Some of $\boldsymbol{\delta}_{1}^{\circ}, \boldsymbol{\delta}_{2}^{\circ}, \ldots, \boldsymbol{\delta}_{m}^{\circ}$ may be equal as can be seen from an example of a normal mixture. So, if $\boldsymbol{X}$ has the finite mixture (1.1), then the pdf (1.2) of $Y$ can be rewritten as a finite mixture of $m^{*}$ components such as

$$
h\left(y \mid \boldsymbol{c}_{m^{*}}^{*}\right)=\sum_{i=1}^{m^{*}} q_{i}^{\circ} h_{\boldsymbol{\pi}_{i}^{\circ}}(y),
$$

where $\boldsymbol{c}_{m^{*}}^{*}=\left(q_{1}^{\circ}, q_{2}^{\circ}, \ldots, q_{m^{*}}^{\circ} ; \boldsymbol{\pi}_{1}^{\circ}, \boldsymbol{\pi}_{2}^{\circ}, \ldots, \boldsymbol{\pi}_{m^{*}}^{\circ}\right)$. Of course, if all of $\boldsymbol{\delta}_{1}^{\circ}, \boldsymbol{\delta}_{2}^{\circ}, \ldots, \boldsymbol{\delta}_{m}^{\circ}$ are different, then $m^{*}=m$ holds. 
Putting $Y_{j}=\boldsymbol{a}^{\prime} \boldsymbol{X}_{j}+\rho$, then $\left(Y_{1}, Y_{2}, \ldots, Y_{n}\right)$ can be considered an independent random sample from the distribution (2.2) by the argument above. As a preliminary, to give an estimator of $m$, we first construct an estimator of $m^{*}$ of (2.2) on the basis of $\left(Y_{1}, Y_{2}, \ldots, Y_{n}\right)$. In order to do this, we assume that the cumulative distribution function $F_{\boldsymbol{\delta}}(y)$ corresponding to $h_{\boldsymbol{\delta}}(y)$ is continuous in $\boldsymbol{\delta}$ on $\Delta(\boldsymbol{a}, \rho)$ for each $y$. Putting $\boldsymbol{c}_{\ell}=\left(q_{1}, q_{2}, \ldots, q_{\ell} ; \boldsymbol{\delta}_{1}, \boldsymbol{\delta}_{2}, \cdots, \boldsymbol{\delta}_{\ell}\right)$, let us define parameter spaces by

$$
\begin{array}{r}
\mathcal{B}(\ell \mid \boldsymbol{a}, \rho) \\
=\left\{\boldsymbol{c}_{\ell}: \sum_{i=1}^{\ell} q_{i}=1,0 \leq q_{i} \leq 1, \boldsymbol{\delta}_{i} \in \Delta(\boldsymbol{a}, \rho),(i=1,2, \ldots, \ell)\right\}, \\
(\ell=1,2, \ldots) .
\end{array}
$$

Let $\widehat{\boldsymbol{c}}_{\ell, n}=\left(\widehat{q}_{1, n}, \widehat{q}_{2, n}, \ldots, \widehat{q}_{\ell, n} ; \widehat{\boldsymbol{\delta}}_{1, n}, \widehat{\boldsymbol{\delta}}_{2, n}, \ldots, \widehat{\boldsymbol{\delta}}_{\ell, n}\right)$ be any $\boldsymbol{c}_{\ell}$ on $\mathcal{B}(\ell \mid \boldsymbol{a}, \rho)$ which minimizes

$$
S_{n}\left(\boldsymbol{c}_{\ell} \mid \boldsymbol{a}, \rho\right)=\frac{1}{n} \sum_{\alpha=1}^{n}\left\{\sum_{i=1}^{\ell} q_{i} F_{\boldsymbol{\delta}_{i}}\left(Y_{(\alpha)}\right)-\frac{\alpha}{n}\right\}^{2},
$$

where $Y_{(\alpha)}$ is the $\alpha$-th order statistic of $\left(Y_{1}, Y_{2}, \ldots, Y_{n}\right)$. The existence of $\widehat{\boldsymbol{c}}_{\ell, n}$ is guaranteed since $\mathcal{B}(\ell \mid \boldsymbol{a}, \rho)$ is a compact subset of $\mathcal{R}^{\ell\left(1+d_{2}\right)-1}$ and $S_{n}\left(\boldsymbol{c}_{\ell} \mid \boldsymbol{a}, \rho\right)$ continuous in $\boldsymbol{c}_{\ell}$ on $\mathcal{B}(\ell \mid \boldsymbol{a}, \rho)$ from the definition.

Let us now give an estimator of $m^{*}$, which is an improvement of that of Henna (1985), as follows:

$$
\widehat{m}_{n}^{*}=\text { the minimum integer } \ell \text { such that } S_{n}\left(\widehat{\boldsymbol{c}}_{\ell, n} \mid \boldsymbol{a}, \rho\right)<\lambda_{\ell}^{2}(n) / n,
$$

where $\lambda_{\ell}(n) \uparrow \infty, \lambda_{\ell}^{2}(n) / n \rightarrow 0$ as $n \rightarrow \infty$ and $\sum\left\{\lambda_{\ell}^{2}(n) / n\right\} \exp \left\{-2 \lambda_{\ell}^{2}(n)\right\}<\infty$ for each $\ell$. In other words, we first examine whether $\widehat{m}_{n}^{*}=1$ holds or not by $\lambda_{1}(n)$. If the result is $\widehat{m}_{n}^{*}>1$, then we examine whether $\widehat{m}_{n}^{*}=2$ holds or not by $\lambda_{2}(n)$. And so on.

The criterions are desirable to satisfy a condition that the probabilities of $S_{n}\left(\widehat{\boldsymbol{c}}_{\ell, n} \mid \boldsymbol{a}, \rho\right)<\lambda_{\ell}^{2}(n) / n$ and $\lambda_{\ell}^{2}(n) / n<S_{n}\left(\widehat{\boldsymbol{c}}_{\ell+1, n} \mid \boldsymbol{a}, \rho\right)$ are as large as possible according to $m^{*}=\ell$ and $m^{*}=\ell+1$, respectively. Then, for each $n$, the probability $\mathrm{P}\left\{\widehat{m}_{n}^{*}=m^{*}\right\}$ using the method given in this paper is larger than that given by the method in Henna (1985) with a common criterion.

The following lemma can be obtained by a simple modification of the proof of Theorem 4.1 of Henna (1985) under an identifiability condition (Teicher (1963)).

Lemma 2.2. Assume that, for any two finite mixtures $h\left(y \mid \boldsymbol{c}_{\ell_{1}}^{(1)}\right)$ and $h(y \mid$ $\left.\boldsymbol{c}_{\ell_{2}}^{(2)}\right)$, the relationship $h\left(y \mid \boldsymbol{c}_{\ell_{1}}^{(1)}\right)=h\left(y \mid \boldsymbol{c}_{\ell_{2}}^{(2)}\right)$ implies that $\ell_{1}=\ell_{2}$ and $\boldsymbol{c}_{\ell_{1}}^{(1)}=\boldsymbol{c}_{\ell_{2}}^{(2)}$, where $\boldsymbol{c}_{\ell_{1}}^{(1)}=\boldsymbol{c}_{\ell_{2}}^{(2)}$ means for a permutation of parameter labels. Then we have

$$
\mathrm{P}_{\mathcal{A}_{m}^{\circ}}^{(\infty)}\left\{\widehat{m}_{n}^{*}=m^{*} \text { for all } n \text { sufficiently large }\right\}=1 .
$$




\section{An estimator and its asymptotic property in the case of a normal mixture}

Let $\mathcal{F}=\{n(\boldsymbol{x} \mid \boldsymbol{\mu}, \boldsymbol{Z}):(\boldsymbol{\mu}, \boldsymbol{Z}) \in \Theta\}$, where $n(\boldsymbol{x} \mid \boldsymbol{\mu}, \boldsymbol{Z})$ is a $k$-dimensional normal pdf with a mean vector $\boldsymbol{\mu}$ and a variance-covariance matrix $\boldsymbol{Z}$, and $\Theta$ is a compact subset of $\mathcal{R}^{(1 / 2) k(k+1)+k}$. Consider a finite normal mixture

$$
f\left(\boldsymbol{x} \mid \mathcal{A}_{m}^{\circ}\right)=\sum_{i=1}^{m} p_{i}^{\circ} n\left(\boldsymbol{x} \mid \boldsymbol{\mu}_{i}^{\circ}, \boldsymbol{\Psi}_{i}^{\circ}\right)
$$

as a special case of $(1.1)$, where $\mathcal{A}_{m}^{\circ}=\left(p_{1}^{\circ}, p_{2}^{\circ}, \ldots, p_{m}^{\circ} ;\left(\boldsymbol{\mu}_{1}^{\circ}, \boldsymbol{Z}_{1}^{\circ}\right),\left(\boldsymbol{\mu}_{2}^{\circ}, \boldsymbol{Z}_{2}^{\circ}\right), \ldots\right.$, $\left.\left(\boldsymbol{\mu}_{m}^{\circ}, \boldsymbol{Z}_{m}^{\circ}\right)\right)$.

The method here is similar to that of Henna (2005) where a sequence $\left\{\boldsymbol{M}_{\gamma}\right\}$ of orthogonal matrices is considered to give an estimator of $m$ of (3.1). We construct a sequence $\left\{\boldsymbol{a}_{\gamma}\right\}$ of column vectors as follows:

(i) For $\gamma \leq k$, let $\boldsymbol{a}_{\gamma}=(0, \ldots, 0, \underset{(\gamma)}{1}, 0, \ldots, 0)^{\prime}$.

(ii) For $\gamma>k$, let $\boldsymbol{a}_{\gamma}$ be linearly independent of any $k-1$ of $\left\{\boldsymbol{a}_{1}, \boldsymbol{a}_{2}, \ldots, \boldsymbol{a}_{\gamma-1}\right\}$. An example for $k=2$ is given in Section 6 .

We can see that $f_{\boldsymbol{\theta}}(\boldsymbol{x})=n(\boldsymbol{x} \mid \boldsymbol{\mu}, \boldsymbol{Z})$ corresponds to $h_{\boldsymbol{\delta}(\gamma)}(y)=$ $n\left(y \mid \nu^{(\gamma)},\left(\sigma^{(\gamma)}\right)^{2}\right)$ through $Y^{(\gamma)}=\boldsymbol{a}_{\gamma}^{\prime} \boldsymbol{X}+\rho_{\gamma}$ with $\rho_{\gamma}$ a scalar, where $\boldsymbol{\delta}^{(\gamma)}=$ $\left(\nu^{(\gamma)},\left(\sigma^{(\gamma)}\right)^{2}\right)$ with $\nu^{(\gamma)}=\boldsymbol{a}_{\gamma}^{\prime} \boldsymbol{\mu}+\rho_{\gamma}$ and $\left(\sigma^{(\gamma)}\right)^{2}=\boldsymbol{a}_{\gamma}^{\prime} \boldsymbol{\Psi} \boldsymbol{a}_{\gamma}$ (Anderson (2003)). Therefore $\Delta\left(\boldsymbol{a}_{\gamma}, \rho_{\gamma}\right)=\left\{\boldsymbol{\delta}^{(\gamma)}:(\boldsymbol{\mu}, \boldsymbol{Z}) \in \Theta\right\}$ is a compact subset of $\mathcal{R}^{2}$. We can see that $F_{\boldsymbol{\delta}(\gamma)}(y)$ is continuous in $\boldsymbol{\delta}^{(\gamma)}$ on $\Delta\left(\boldsymbol{a}_{\gamma}, \rho_{\gamma}\right)$ for each $y$.

Putting $Y_{j}^{(\gamma)}=\boldsymbol{a}_{\gamma}^{\prime} \boldsymbol{X}_{j}+\rho_{\gamma}$, by the argument of the last section, then $\left(Y_{1}^{(\gamma)}, Y_{2}^{(\gamma)}, \ldots, Y_{n}^{(\gamma)}\right)$ can be considered an independent random sample from the univariate finite normal mixture

$$
h\left(y \mid \boldsymbol{c}_{m(\gamma)}^{(\gamma)}\right)=\sum_{i=1}^{m(\gamma)} q_{i}^{(\gamma)} n\left(y \mid \tau_{i}^{(\gamma)},\left(v_{i}^{(\gamma)}\right)^{2}\right) \quad(\gamma=1,2, \cdots)
$$

where all of $\left(\tau_{1}^{(\gamma)},\left(v_{1}^{(\gamma)}\right)^{2}\right),\left(\tau_{2}^{(\gamma)},\left(v_{2}^{(\gamma)}\right)^{2}\right), \ldots,\left(\tau_{m(\gamma)}^{(\gamma)},\left(v_{m(\gamma)}^{(\gamma)}\right)^{2}\right)$ are different.

Define an estimator $\widehat{m}_{n}^{(\gamma)}$ of $m(\gamma)$ on the basis of $\left(Y_{1}^{(\gamma)}, Y_{2}^{(\gamma)}, \ldots, Y_{n}^{(\gamma)}\right)$ in the same way to (2.5). For estimation problems of finite mixtures, an important matter which should be satisfied is an identifiability condition. Proposition 2 of Yakowitz and Spragins (1968) shows that the class of all finite mixtures of $k$-dimensional normal pdf's is identifiable. So we can obtain the following by Lemma 2.2.

LEMMA 3.1. For any given $\left(\boldsymbol{a}_{\gamma}, \rho_{\gamma}\right)$, we have

$$
\mathrm{P}_{\mathcal{A}_{m}^{\circ}}^{(\infty)}\left\{\widehat{m}_{n}^{(\gamma)}=m(\gamma) \text { for all } n \text { sufficiently large }\right\}=1 \text {. }
$$

Unfortunately, the number $m(\gamma)$ is not necessarily equal to $m$ as mentioned before. However, by Lemma A.1, we can obtain the following theorem which gives a condition to hold $m(\gamma)=m$. 
THEOREM 3.1. Assume that $\boldsymbol{X}$ has the finite mixture (3.1) with different $\boldsymbol{\mu}_{1}^{\circ}, \boldsymbol{\mu}_{2}^{\circ}, \ldots, \boldsymbol{\mu}_{m}^{\circ}$. If $m(m-1)(k-1)<2 s$, then there exists $\left(\boldsymbol{a}_{\gamma}, \rho_{\gamma}\right)(\gamma \leq s)$ such that $Y^{(\gamma)}=\boldsymbol{a}_{\gamma}^{\prime} \boldsymbol{X}+\rho_{\gamma}$ also has the finite mixture of $m$ one-dimensional normal pdf's such as

$$
h\left(y \mid \boldsymbol{c}_{m}^{(\gamma)}\right)=\sum_{i=1}^{m} p_{i}^{\circ} n\left(y \mid \nu_{i}^{(\gamma)},\left(\sigma_{i}^{(\gamma)}\right)^{2}\right),
$$

where $\boldsymbol{c}_{m}^{(\gamma)}=\left(p_{1}^{\circ}, p_{2}^{\circ}, \ldots, p_{m}^{\circ} ;\left(\nu_{1}^{(\gamma)},\left(\sigma_{1}^{(\gamma)}\right)^{2}\right),\left(\nu_{2}^{(\gamma)},\left(\sigma_{2}^{(\gamma)}\right)^{2}\right), \ldots,\left(\nu_{m}^{(\gamma)},\left(\sigma_{m}^{(\gamma)}\right)^{2}\right)\right)$ with $\nu_{i}^{(\gamma)}=\boldsymbol{a}_{\gamma}^{\prime} \boldsymbol{\mu}_{i}^{\circ}+\rho_{\gamma}$ and $\left(\sigma_{i}^{(\gamma)}\right)^{2}=\boldsymbol{a}_{\gamma}^{\prime} \boldsymbol{\xi}_{i}^{\circ} \boldsymbol{a}_{\gamma}(i=1,2, \ldots, m)$, and all of $\left(\nu_{1}^{(\gamma)},\left(\sigma_{1}^{(\gamma)}\right)^{2}\right),\left(\nu_{2}^{(\gamma)},\left(\sigma_{2}^{(\gamma)}\right)^{2}\right), \ldots,\left(\nu_{m}^{(\gamma)},\left(\sigma_{m}^{(\gamma)}\right)^{2}\right)$ are different.

An important point of the theorem above is that, even if we have no knowledge other than a fact that $\boldsymbol{X}$ has a multivariate finite normal mixture with different mean vectors, we can certainly obtain a univariate random variable which has a finite normal mixture preserving the number of components by constructing $Y^{(1)}, Y^{(2)}, \ldots$ sequentially.

Hence, if $m(m-1)(k-1)<2 s$, then at least one of $m(1), m(2), \ldots, m(s)$ equals to $m$, and $m(\gamma) \leq m$ holds for any $\gamma$. Therefore, by putting

$$
\widehat{m}_{n}(s)=\max _{1 \leq \gamma \leq s} \widehat{m}_{n}^{(\gamma)} \quad(s=1,2, \ldots),
$$

we have the following.

LEMMA 3.2. Assume that all of $\boldsymbol{\mu}_{1}^{\circ}, \boldsymbol{\mu}_{2}^{\circ}, \ldots, \boldsymbol{\mu}_{m}^{\circ}$ are different and $m(m-$ $1)(k-1)<2 s$ holds. Then we have

$$
\mathrm{P}_{\mathcal{A}_{m}^{\circ}}^{(\infty)}\left\{\widehat{m}_{n}(s)=m \text { for all } n \text { sufficiently large }\right\}=1 .
$$

But, as $m$ is unknown, we cannot know at any given step $s$ whether the condition $m(m-1)(k-1)<2 s$ holds or not. Then we cannot know when stopping the algorithm to give $\widehat{m}_{n}(s)$ which actually satisfies (3.6). However, if we construct $\widehat{m}_{n}(1), \widehat{m}_{n}(2), \ldots$ sequentially, then we can obtain $\widehat{m}_{n}(s)$ which satisfies $m(m-1)(k-1)<2 s$ and consequently (3.6) before long. Referring to this, we give two lemmas which suggest a criterion to make sure that $m(m-1)(k-1)<2 s$ holds. First, by $m(\gamma) \leq m$ for any $\gamma$, we can obtain the following from Lemma 3.1 .

LEMMA 3.3. For any given positive integer $s_{1}$, we have

$$
\mathrm{P}_{\mathcal{A}_{m}^{\circ}}^{(\infty)}\left\{\widehat{m}_{n}(s) \leq m\left(s=1,2, \ldots, s_{1}\right) \text { for all } n \text { sufficiently large }\right\}=1 .
$$

Next, let $s_{\circ}$ be the minimum positive integer $s$ such as $m(m-1)(k-1)<2 s$. Then the following is an immediate consequence of Lemma 3.2. 
Lemma 3.4. Assume that all of $\boldsymbol{\mu}_{1}^{\circ}, \boldsymbol{\mu}_{2}^{\circ}, \ldots, \boldsymbol{\mu}_{m}^{\circ}$ are different. Then, for any given positive integer $s_{1}$ such as $s_{\circ} \leq s_{1}$, we have

$$
\mathrm{P}_{\mathcal{A}_{m}^{\circ}}^{(\infty)}\left\{\widehat{m}_{n}(s)=m\left(s=s_{\circ}, s_{\circ}+1, \ldots, s_{1}\right) \text { for all } n \text { sufficiently large }\right\}=1 \text {. }
$$

As can be seen from the definition, $\widehat{m}_{n}(s)$ is monotone increasing in $s$ for any given $\left(\boldsymbol{X}_{1}, \boldsymbol{X}_{2}, \ldots, \boldsymbol{X}_{n}\right)$. In addition, if all of $\boldsymbol{\mu}_{1}^{\circ}, \boldsymbol{\mu}_{2}^{\circ}, \ldots, \boldsymbol{\mu}_{m}^{\circ}$ are different, it can be considered that $\widehat{m}_{n}(s) \leq m$ when $s \leq s_{\circ}-1$ and $\widehat{m}_{n}(s)=m$ when $s_{\circ} \leq s \leq s_{1}$ for sufficiently large $n$ by Lemmas 3.3 and 3.4, respectively. Hence, it can be considered that the increasing sequence $\widehat{m}_{n}(1), \widehat{m}_{n}(2), \ldots$ may become invariant soon for sufficiently large $n$. So, when the sequence becomes invariant, it can be considered that $m(m-1)(k-1)<2 s$ holds and the invariant value equals to $m$.

Taking these into account, we now give an estimator of $m$ as follows:

$$
\widehat{m}_{n}=\widehat{m}_{n}\left(s_{\circ}^{*}\right),
$$

where $s_{\circ}^{*}$ is the minimum positive integer $s$ such as $\widehat{m}_{n}(s)=\widehat{m}_{n}\left(s+s_{1}^{*}\right)$ with $s_{1}^{*}$ a given positive integer.

If the same value succeeds $s_{1}^{*}+1$ steps on $\left\{\widehat{m}_{n}(1), \widehat{m}_{n}(2), \ldots\right\}$, then the algorithm is terminated. It is natural to regard the same value as an estimate of $m$. The existence of $\widehat{m}_{n}$ is guaranteed with probability one, for sufficiently large $n$, by the last lemma. It can be seen that $\widehat{m}_{n}$ is given without any knowledge about $m$ other than a fact that $m$ is finite. If $s_{1}^{*}$ is sufficiently large, then $m(m-1)(k-1)<2\left(s_{\circ}^{*}+s_{1}^{*}\right)$ holds. Accordingly, the following can be easily obtained from the last two lemmas.

Theorem 3.2. Assume that all of $\boldsymbol{\mu}_{1}^{\circ}, \boldsymbol{\mu}_{2}^{\circ}, \ldots, \boldsymbol{\mu}_{m}^{\circ}$ are different. Then, for sufficiently large $s_{1}^{*}$, we have

$$
\mathrm{P}_{\mathcal{A}_{m}^{\circ}}^{(\infty)}\left\{\widehat{m}_{n}=m \text { for all } n \text { sufficiently large }\right\}=1 .
$$

The theorem above states the asymptotic behavior using $\widehat{m}_{n}$. No further reference to the $m(m-1)(k-1)<2 s$ condition is needed. In fact, as $m$ is finite, we assure that there exists an integer $s_{1}^{*}$ such that $m(m-1)(k-1)<2\left(s_{\circ}^{*}+s_{1}^{*}\right)$, so that at least one of $\left\{m(\gamma): \gamma=1,2, \ldots, s_{\circ}^{*}+s_{1}^{*}\right\}$ is equal to $m$ by Theorem 3.1 .

For implementing the algorithm, a problem is to determine the number of steps of the same value which will succeed on $\left\{\widehat{m}_{n}(1), \widehat{m}_{n}(2), \ldots\right\}$. And here, we have no way to do that except to consider an upper bound using applicable arguments or to define a priori a length $s_{1}^{*}=5$ for example (but there may also be linear algebra considerations that can lead to sufficient conditions).

Remark 3.1. The method here can not be applied to the case where some of the components have the same mean vector but different variance-covariance 
matrices, because the number of linear combinations which preserve the number of components is not necessarily finite.

\section{Another property of the estimator in the case of a normal mixture}

We show here that $\widehat{m}_{n}$ is independent of $\left(\left\|\boldsymbol{a}_{\gamma}\right\|, \rho_{\gamma}\right)$ for any $\left(\boldsymbol{a}_{\gamma}, \rho_{\gamma}\right)$, where $\left\|\boldsymbol{a}_{\gamma}\right\|^{2}=\boldsymbol{a}_{\gamma}^{\prime} \boldsymbol{a}_{\gamma}$. For this purpose, replacing $\left(\boldsymbol{a}_{\gamma}, \rho_{\gamma}\right)$ by $(\boldsymbol{a}, \rho)$ for convenience sake, define $\Delta(\boldsymbol{a}, \rho), \mathcal{B}(\ell \mid \boldsymbol{a}, \rho), S_{n}\left(\boldsymbol{c}_{\ell} \mid \boldsymbol{a}, \rho\right)$ and $\widehat{\boldsymbol{c}}_{\ell, n}=\left(\widehat{q}_{1, n}, \widehat{q}_{2, n}, \ldots, \widehat{q}_{\ell, n}\right.$; $\left.\left(\widehat{\nu}_{1, n},\left(\widehat{\sigma}_{1, n}\right)^{2}\right),\left(\widehat{\nu}_{2, n},\left(\widehat{\sigma}_{2, n}\right)^{2}\right), \ldots,\left(\widehat{\nu}_{\ell, n},\left(\widehat{\sigma}_{\ell, n}\right)^{2}\right)\right)$ in similar ways to those of Section 2, respectively. The following gives a property of the minimum value of $S_{n}$.

LEMMA 4.1. The minimum value $S_{n}\left(\widehat{\boldsymbol{c}}_{\ell, n} \mid \boldsymbol{a}, \rho\right)$ is independent of $(\|\boldsymbol{a}\|, \rho)$ for any $\ell$.

Proof. Let $\beta(>0)$ be a scalar. Assume that $S_{n}\left(\boldsymbol{c}_{\ell} \mid \beta \boldsymbol{a}, \rho\right)$ and $S_{n}\left(\boldsymbol{c}_{\ell} \mid\right.$ $\boldsymbol{a}, 0)$ attain the minimum values at $\widehat{\boldsymbol{c}}_{\ell, n}^{*} \in \mathcal{B}(\ell \mid \beta \boldsymbol{a}, \rho)$ and $\widehat{\boldsymbol{c}}_{\ell, n} \in \mathcal{B}(\ell \mid \boldsymbol{a}, 0)$, respectively. Let $Y_{j}^{*}=(\beta \boldsymbol{a})^{\prime} \boldsymbol{X}_{j}+\rho$ and $Y_{j}=\boldsymbol{a}^{\prime} \boldsymbol{X}_{j}(j=1,2, \ldots, n)$. Then we have $Y_{(\alpha)}^{*}=\beta Y_{(\alpha)}+\rho(\alpha=1,2, \ldots, n)$, where $Y_{(\alpha)}^{*}$ and $Y_{(\alpha)}$ are the $\alpha$-th order statistics of $\left(Y_{1}^{*}, Y_{2}^{*}, \ldots, Y_{n}^{*}\right)$ and $\left(Y_{1}, Y_{2}, \ldots, Y_{n}\right)$, respectively. From the definition, we have

$$
\Delta(\boldsymbol{a}, 0)=\left\{\left(\nu, \sigma^{2}\right): \nu=\boldsymbol{a}^{\prime} \boldsymbol{\mu}, \sigma^{2}=\boldsymbol{a}^{\prime} \boldsymbol{Z} \boldsymbol{a},(\boldsymbol{\mu}, \boldsymbol{\Psi}) \in \Theta\right\}
$$

and

$$
\Delta(\beta \boldsymbol{a}, \rho)=\left\{\left(\nu^{*},\left(\sigma^{*}\right)^{2}\right): \nu^{*}=\beta \nu+\rho,\left(\sigma^{*}\right)^{2}=\beta^{2} \sigma^{2},\left(\nu, \sigma^{2}\right) \in \Delta(\boldsymbol{a}, 0)\right\} .
$$

Hence there exists $\left(\nu_{i}, \sigma_{i}^{2}\right) \in \Delta(\boldsymbol{a}, 0)$ such as $\left(\widehat{\nu}_{i, n}^{*},\left(\widehat{\sigma}_{i, n}^{*}\right)^{2}\right)=\left(\beta \nu_{i}+\rho, \beta^{2} \sigma_{i}^{2}\right)$, where $\widehat{\boldsymbol{c}}_{\ell, n}^{*}=\left(\widehat{q}_{1, n}^{*}, \widehat{q}_{2, n}^{*}, \ldots, \widehat{q}_{\ell, n}^{*} ;\left(\widehat{\nu}_{1, n}^{*},\left(\widehat{\sigma}_{1, n}^{*}\right)^{2}\right),\left(\widehat{\nu}_{2, n}^{*},\left(\widehat{\sigma}_{2, n}^{*}\right)^{2}\right), \ldots,\left(\widehat{\nu}_{\ell, n}^{*},\left(\widehat{\sigma}_{\ell, n}^{*}\right)^{2}\right)\right)$. Substituting $y=\beta z+\rho$, then we have

$$
\begin{aligned}
S_{n}\left(\widehat{\boldsymbol{c}}_{\ell, n}^{*} \mid \beta \boldsymbol{a}, \rho\right) & =\frac{1}{n} \sum_{\alpha=1}^{n}\left\{\sum_{i=1}^{\ell} \widehat{q}_{i, n}^{*} \int_{-\infty}^{Y_{(\alpha)}^{*}} n\left(y \mid \widehat{\nu}_{i, n}^{*},\left(\widehat{\sigma}_{i, n}^{*}\right)^{2}\right) d y-\frac{\alpha}{n}\right\}^{2} \\
& =\frac{1}{n} \sum_{\alpha=1}^{n}\left\{\sum_{i=1}^{\ell} \widehat{q}_{i, n}^{*} \int_{-\infty}^{Y_{(\alpha)}} n\left(z \mid \nu_{i}, \sigma_{i}^{2}\right) d z-\frac{\alpha}{n}\right\}^{2} \\
& =S_{n}\left(\boldsymbol{c}_{\ell} \mid \boldsymbol{a}, 0\right) \\
& \geq S_{n}\left(\widehat{\boldsymbol{c}}_{\ell, n} \mid \boldsymbol{a}, 0\right)
\end{aligned}
$$

where $\boldsymbol{c}_{\ell}=\left(\widehat{q}_{1, n}^{*}, \widehat{q}_{2, n}^{*}, \ldots, \widehat{q}_{\ell, n}^{*} ;\left(\nu_{1}, \sigma_{1}^{2}\right),\left(\nu_{2}, \sigma_{2}^{2}\right), \ldots,\left(\nu_{\ell}, \sigma_{\ell}^{2}\right)\right)$. The last inequality holds because $\boldsymbol{c}_{\ell} \in \mathcal{B}(\ell \mid \boldsymbol{a}, 0)$. In a similar way, we have

$$
S_{n}\left(\widehat{\boldsymbol{c}}_{\ell, n}^{*} \mid \beta \boldsymbol{a}, \rho\right) \leq S_{n}\left(\widehat{\boldsymbol{c}}_{\ell, n} \mid \boldsymbol{a}, 0\right)
$$

Accordingly, we have

$$
S_{n}\left(\widehat{\boldsymbol{c}}_{\ell, n}^{*} \mid \beta \boldsymbol{a}, \rho\right)=S_{n}\left(\widehat{\boldsymbol{c}}_{\ell, n} \mid \boldsymbol{a}, 0\right)
$$


which shows that the minimum value of $S_{n}$ is invariant with respect to $(\beta, \rho)$ for any $\ell$. This completes the proof.

The lemma above shows that $\widehat{m}_{n}^{(\gamma)}$ is independent of $\left(\left\|\boldsymbol{a}_{\gamma}\right\|, \rho_{\gamma}\right)$ for any $\gamma$. Hence, from the definition, we have the following.

THEOREM 4.1. $\widehat{m}_{n}$ is independent of $\left(\left\|\boldsymbol{a}_{\gamma}\right\|, \rho_{\gamma}\right)$ for any $\gamma$.

\section{An estimator and its properties in the case of a known finite family of normal pdf's}

We consider here $\mathcal{F}=\{n(\boldsymbol{x} \mid \boldsymbol{\mu}, \boldsymbol{Z}):(\boldsymbol{\mu}, \boldsymbol{Z}) \in \Theta\}$ with a known finite set $\Theta$ of $L$ elements. Let $\left\{\boldsymbol{a}_{\gamma}\right\}$ be that of Section 3. Defining $\Delta\left(\boldsymbol{a}_{\gamma}, \rho_{\gamma}\right), \mathcal{B}\left(\ell \mid \boldsymbol{a}_{\gamma}, \rho_{\gamma}\right)$, $S_{n}\left(\boldsymbol{c}_{\ell, n} \mid \boldsymbol{a}_{\gamma}, \rho_{\gamma}\right)$ and $\widehat{\boldsymbol{c}}_{\ell, n}$ for $\left(\boldsymbol{a}_{\gamma}, \rho_{\gamma}\right)$ in similar ways to those of Section 2, respectively, we give an estimator of $m(\gamma)$ as follows:

$$
\widehat{m}_{n}^{(\gamma)}=\left\{\begin{array}{l}
\text { the minimum integer } \ell\left(\leq L_{\gamma}-1\right) \text { such as } \\
S_{n}\left(\widehat{\boldsymbol{c}}_{\ell, n} \mid \boldsymbol{a}_{\gamma}, \rho_{\gamma}\right)<\lambda_{\ell}^{2}(n) / n \\
\text { or } \\
L_{\gamma} \text { if } S_{n}\left(\widehat{\boldsymbol{c}}_{\ell, n} \mid \boldsymbol{a}_{\gamma}, \rho_{\gamma}\right) \geq \lambda_{\ell}^{2}(n) / n \text { for all } \ell\left(\leq L_{\gamma}-1\right)
\end{array}\right.
$$

where $\lambda_{\ell}$ 's are those of (2.5) and $L_{\gamma}$ is the number of elements of $\Delta\left(\boldsymbol{a}_{\gamma}, \rho_{\gamma}\right)$. Defining $\widehat{m}_{n}$ in the same way to that of (3.9), then, in a similar way to Theorem 3.2 , we can prove the following.

TheOREM 5.1. Assume that all of $\boldsymbol{\mu}_{1}^{\circ}, \boldsymbol{\mu}_{2}^{\circ}, \ldots, \boldsymbol{\mu}_{m}^{\circ}$ are different. Then, for sufficiently large $s_{1}^{*}$, we have

$$
\mathrm{P}_{\mathcal{A}_{m}^{\circ}}^{(\infty)}\left\{\widehat{m}_{n}=m \text { for all } n \text { sufficiently large }\right\}=1 .
$$

Furthermore, we can obtain the following by the definition of $\widehat{m}_{n}$ and Lemma 4.1 .

THEOREM 5.2. $\widehat{m}_{n}$ is independent of $\left(\left\|\boldsymbol{a}_{\gamma}\right\|, \rho_{\gamma}\right)$ for any $\gamma$.

\section{Some simulation results}

Now we give some simulation results for Theorem 5.1. The family of pdf's considered here is $\mathcal{F}=\left\{n\left(\boldsymbol{x} \mid \boldsymbol{\mu}_{i}, I\right): i=1,2,3,4\right\}$, where $\boldsymbol{\mu}_{1}=(0,0)^{\prime}$, $\boldsymbol{\mu}_{2}=(0,4)^{\prime}, \boldsymbol{\mu}_{3}=(4,4)^{\prime}, \boldsymbol{\mu}_{4}=(4,8)^{\prime}$ and $I$ is an identity matrix. In order to obtain univariate independent random samples, considering that $S_{n}\left(\widehat{\boldsymbol{c}}_{\ell, n} \mid \boldsymbol{a}_{\gamma}, \rho_{\gamma}\right)$ depends only on the direction coefficients of $\boldsymbol{a}_{\gamma}$ by Lemma 4.1, we tried with $\boldsymbol{a}_{1}=$ $(1,0)^{\prime}, \boldsymbol{a}_{2}=(0,1)^{\prime}, \boldsymbol{a}_{3}=(0.717106,0.696964)^{\prime}, \boldsymbol{a}_{4}=(-0.696964,0.717106)^{\prime}$, $\boldsymbol{a}_{5}=\left(\cos \left(\frac{5 \pi}{8}\right), \sin \left(\frac{5 \pi}{8}\right)\right)^{\prime}, \boldsymbol{a}_{6}=\left(\cos \left(\frac{3 \pi}{8}\right), \sin \left(\frac{3 \pi}{8}\right)\right)^{\prime}, \boldsymbol{a}_{7}=\left(\cos \left(\frac{\pi}{8}\right), \sin \left(\frac{\pi}{8}\right)\right)^{\prime}$ and $\boldsymbol{a}_{8}=\left(\cos \left(-\frac{\pi}{8}\right), \sin \left(-\frac{\pi}{8}\right)\right)^{\prime}$ with $\rho_{\gamma}=0(\gamma=1,2, \ldots, 8)$. For criterions, $\lambda_{1}^{2}(n) / n=$ $(\log \log n)^{2} / n, \lambda_{2}^{2}(n) / n=(\log \log n)^{2} / 5 n$ and $\lambda_{3}^{2}(n) / n=(\log \log n)^{2} / 10 n$ were used. 
Table 1. Percentages of exact estimate of $m$ for equal mixing ratios.

\begin{tabular}{ccccc}
\hline & $\begin{array}{c}\text { a single } \\
\text { component }\end{array}$ & $\begin{array}{c}\text { two } \\
\text { components }\end{array}$ & $\begin{array}{c}\text { three } \\
\text { components }\end{array}$ & $\begin{array}{c}\text { four } \\
\text { components }\end{array}$ \\
\hline$n=200$ & 100 & 100 & 99.92 & 95.40 \\
\hline$n=300$ & 100 & 100 & 100 & 99.84 \\
\hline$n=400$ & 100 & 100 & 100 & 99.98 \\
\hline$n=500$ & 100 & 100 & 100 & 100 \\
\hline
\end{tabular}

Table 2. Percentages of exact estimate of $m$ for different mixing ratios.

\begin{tabular}{cccc}
\hline & $\begin{array}{c}\text { two } \\
\text { components }\end{array}$ & $\begin{array}{c}\text { three } \\
\text { components }\end{array}$ & $\begin{array}{c}\text { four } \\
\text { components }\end{array}$ \\
\hline$n=200$ & 100 & 98.90 & 78.00 \\
\hline$n=300$ & 100 & 100 & 96.44 \\
\hline$n=400$ & 100 & 100 & 99.68 \\
\hline$n=500$ & 100 & 100 & 100 \\
\hline
\end{tabular}

Using random numbers produced by The Institute of Statistical Mathematics, 5000 bivariate samples of sizes $n=200,300,400$ and 500 were generated from seven types of mixture of $\mathcal{F}$, respectively. When the same value succeeded 5 steps on $\left\{\widehat{m}_{n}(1), \widehat{m}_{n}(2), \ldots, \widehat{m}_{n}(8)\right\}$ for each sample, the algorithm was stopped and the value was regarded as an estimate of $m$. In other cases, the algorithm also was stopped with 8 steps but it was considered that an estimation failed.

Table 1 gives us the percentages of the exact estimates of $m$ by $\widehat{m}_{n}$ for a single normal pdf $n\left(\boldsymbol{x} \mid \boldsymbol{\mu}_{1}, I\right)$, for two components $(1 / 2) n\left(\boldsymbol{x} \mid \boldsymbol{\mu}_{1}, I\right)+(1 / 2) n\left(\boldsymbol{x} \mid \boldsymbol{\mu}_{2}, I\right)$, for three components $(1 / 3) n\left(\boldsymbol{x} \mid \boldsymbol{\mu}_{1}, I\right)+(1 / 3) n\left(\boldsymbol{x} \mid \boldsymbol{\mu}_{2}, I\right)+(1 / 3) n\left(\boldsymbol{x} \mid \boldsymbol{\mu}_{3}, I\right)$ and for four components $(1 / 4) n\left(\boldsymbol{x} \mid \boldsymbol{\mu}_{1}, I\right)+(1 / 4) n\left(\boldsymbol{x} \mid \boldsymbol{\mu}_{2}, I\right)+(1 / 4) n(\boldsymbol{x} \mid$ $\left.\boldsymbol{\mu}_{3}, I\right)+(1 / 4) n\left(\boldsymbol{x} \mid \boldsymbol{\mu}_{4}, I\right)$, respectively.

Table 2 gives us the same as the above for two components $(2 / 5) n\left(\boldsymbol{x} \mid \boldsymbol{\mu}_{1}, I\right)+$ $(3 / 5) n\left(\boldsymbol{x} \mid \boldsymbol{\mu}_{2}, I\right)$, for three components $(3 / 10) n\left(\boldsymbol{x} \mid \boldsymbol{\mu}_{1}, I\right)+(3 / 10) n\left(\boldsymbol{x} \mid \boldsymbol{\mu}_{2}, I\right)+$ $(4 / 10) n\left(\boldsymbol{x} \mid \boldsymbol{\mu}_{3}, I\right)$ and for four components $(2 / 10) n\left(\boldsymbol{x} \mid \boldsymbol{\mu}_{1}, I\right)+(2 / 10) n(\boldsymbol{x} \mid$ $\left.\boldsymbol{\mu}_{2}, I\right)+(3 / 10) n\left(\boldsymbol{x} \mid \boldsymbol{\mu}_{3}, I\right)+(3 / 10) n\left(\boldsymbol{x} \mid \boldsymbol{\mu}_{4}, I\right)$, respectively.

\section{Discussion}

We compare Henna's methods (2005) with those in this paper. First, for $\left\{\boldsymbol{a}_{\gamma}\right\}$ to give the linear combinations, $\left\|\boldsymbol{a}_{\gamma}\right\|=1$ and $\boldsymbol{a}_{\beta k+1}, \boldsymbol{a}_{\beta k+2}, \ldots, \boldsymbol{a}_{\beta k+k}$, which give $M_{\beta+1}(\beta=0,1,2, \ldots)$, are needed to be orthogonal in Henna (2005). However, these conditions are not necessary in this paper, so that the construction of the sequence becomes easy. For $\widehat{m}_{n}(s), k s$ and $s$ vectors are needed in Henna (2005) and in this paper, respectively. So, it can be expected that the number of vectors to get the value of $\widehat{m}_{n}$ in this paper is smaller than that in Henna (2005). Furthermore, there is the case where a computation to obtain $S_{n}\left(\widehat{\boldsymbol{c}}_{\ell, n} \mid \boldsymbol{a}_{\gamma}, \rho_{\gamma}\right)$ becomes easy with an adjustment of length $\left\|\boldsymbol{a}_{\gamma}\right\|$ allowed by Lemma 4.1. So, it becomes easy to get $\widehat{m}_{n}$. 
Second, a common criterion $\lambda^{2}(n) / n$ is used in Henna (2005). On the other hand, the criterions $\lambda_{\ell}^{2}(n) / n(\ell=1,2, \ldots)$ in this paper are given in connection with the number of components $m^{*}$ of $(2.2)$ as mentioned in Section 2. Hence, it can be expected that the probability $\mathrm{P}\left\{\widehat{m}_{n}=m\right\}$ by the methods in this paper is larger than that by the methods in Henna (2005) though it is impossible to give the proof because the vectors used in both papers are not necessarily equal as can be seen from the construction. In the simulation, $\lambda_{\ell}(n)(\ell=1,2$ and 3$)$ were given by trial and error for $m^{*}=1,2,3$ and 4 . It seems that the simulation results show that the criterions are effective when the mixing ratios are nearly equal values. However, a question of which is the optimum in the class of $\lambda_{\ell}$ 's satisfying the condition of (2.5) is worthy of further research.

Next is how to construct $\left\{\boldsymbol{a}_{\gamma}\right\}$. It is natural to use the information of $\boldsymbol{\mu}_{1}^{\circ}, \boldsymbol{\mu}_{2}^{\circ}, \cdots, \boldsymbol{\mu}_{m}^{\circ}$ if it is obtained. Otherwise, a procedure is proposed for $k=2$ in the last section. For $k \geq 3$, a similar method may be adopted but it becomes more complex as $k$ becomes larger. So, the question of which is the optimum in the class of column vectors is worthy of further research.

Finally, as an inequality $\left(\nu_{1}-\nu_{2}\right)^{2} \leq \sum_{i=1}^{k}\left(\mu_{i 1}-\mu_{i 2}\right)^{2}$ holds, a distance between $n\left(y \mid \nu_{1}, 1\right)$ and $n\left(y \mid \nu_{2}, 1\right)$ is smaller than that between $n\left(\boldsymbol{x} \mid \boldsymbol{\mu}_{1}, I\right)$ and $n\left(\boldsymbol{x} \mid \boldsymbol{\mu}_{2}, I\right)$, where $\nu_{j}=\boldsymbol{a}^{\prime} \boldsymbol{\mu}_{j}(j=1,2)$ with $\|\boldsymbol{a}\|=1$. So, there exists a case where the detection of a distinction between $n\left(y \mid \nu_{1}, 1\right)$ and $n\left(y \mid \nu_{2}, 1\right)$ becomes more difficult than that between $n\left(\boldsymbol{x} \mid \boldsymbol{\mu}_{1}, I\right)$ and $n\left(\boldsymbol{x} \mid \boldsymbol{\mu}_{2}, I\right)$. Hence it can be guessed that the estimation by $\widehat{m}_{n}$ tends to give an underestimate. In fact, in the simulation for $(3 / 10) n\left(\boldsymbol{x} \mid \boldsymbol{\mu}_{1}, I\right)+(3 / 10) n\left(\boldsymbol{x} \mid \boldsymbol{\mu}_{2}, I\right)+(4 / 10) n\left(\boldsymbol{x} \mid \boldsymbol{\mu}_{3}, I\right)$ with 200 sample size, for example, the percentages of $\widehat{m}_{n}=2,3,4$ and failure were $0.62,98.9,0.0$ and 0.48 , respectively. Thus, it is necessary to produce a method to modify the bias which is considered to have an origin in the dimension reduction.

\section{Appendix A}

We here give a linear algebra consideration which leads to the construction of a univariate random variable $Y$, preserving the number of components $m$, by transforming $\boldsymbol{X}$ which has the pdf (3.1). Let $\left\{\boldsymbol{a}_{\gamma}\right\}$ be a sequence given in Section 3. As the number of $\boldsymbol{a}_{\gamma}$ 's orthogonal to $\boldsymbol{\mu}_{i}-\boldsymbol{\mu}_{j}(\neq \mathbf{0})$ is $k-1$ at most, the number of $\boldsymbol{a}_{\gamma}$ 's such as $\boldsymbol{a}_{\gamma}^{\prime} \boldsymbol{\mu}_{i}=\boldsymbol{a}_{\gamma}^{\prime} \boldsymbol{\mu}_{j}(i, j=1,2, \ldots, m, i<j)$ is $(k-1)\left(\begin{array}{c}m \\ 2\end{array}\right)$ at most. Accordingly, we have the following.

Lemma A.1. For any different $\boldsymbol{\mu}_{1}, \boldsymbol{\mu}_{2}, \ldots, \boldsymbol{\mu}_{m}$, if $m(m-1)(k-1)<2 s$, then there exists $\boldsymbol{a}_{\gamma}(\gamma \leq s)$ such that all of $\boldsymbol{a}_{\gamma}^{\prime} \boldsymbol{\mu}_{1}, \boldsymbol{a}_{\gamma}^{\prime} \boldsymbol{\mu}_{2}, \ldots, \boldsymbol{a}_{\gamma}^{\prime} \boldsymbol{\mu}_{m}$ are different.

\section{Acknowledgements}

The author wishes to express his hearty thanks to the referees for their valuable comments and suggestions.

\section{REFERENCES}

Anderson, T. W. (2003). An Introduction to Multivariate Statistical Analysis, Third Edition, Wiley, New York. 
Billingsley, P. (1986). Probability and Measure, Second Edition, Wiley, New York.

Chen, J. and Kalbfleisch, J. D. (1996). Penalized minimum-distance estimates in finite mixture models, The Canadian Journal of Statistics, 24, 167-175.

Chen, H., Chen, J. and Kalbfleisch, J. D. (2001). A modified likelihood ratio test for homogeneity in finite mixture models, Journal of the Royal Statistical Society: Series B, 63, $19-29$.

Feng, Z. D. and McCulloch, C. E. (1994). On the likelihood ratio test statistic for the number of components in a normal mixture with unequal variance, Biometrics, 50, 1158-1162.

Garel, B. (2001). Likelihood ratio test for univariate Gaussian mixture, Journal of Statistical Planning and Inference, 96, 325-350.

Henna, J. (1985). On estimating of the number of constituents of a finite mixture of continuous distributions, Annals of the Institute of Statistical Mathematics, 37, 235-240.

Henna, J. (2005). Estimation of the number of components of finite mixtures of multivariate distributions, Annals of the Institute of Statistical Mathematics, 57, 655-664.

Keribin, C. (2000). Consistent estimation of the order of mixture models, Sankhya, 62, 49-66.

McLachlan, G. J. and Basford, K. E. (1988). Mixture Models, Marcel Dekker.

McLachlan, G. and Peel, D. (2000). Finite Mixture Models, Wiley, New York.

Richardson, S. and Green, P. J. (1997). On Bayesian analysis of mixtures with an unknown number of components, Journal of the Royal Statistical Society: Series B, 59, 731-792.

Roeder, K. (1994). A graphical technique for determining the number of components in a mixture of normals, Journal of the American Statistical Association, 89, 487-495.

Teicher, H. (1963). Identifiability of finite mixtures, The Annals of Mathematical Statistics, 34, $1265-1269$.

Titterington, D. M. (1990). Some recent research in the analysis of mixture distributions, Statistics, 21, 619-641.

Titterington, D. M., Smith, A. F. M. and Makov, U. E. (1985). Statistical Analysis of Finite Mixture Distributions, Wiley, New York.

Yakowitz, S. J. and Spragins, J. D. (1968). On the identifiability of finite mixtures, The Annals of Mathematical Statistics, 39, 209-214. 